\title{
ON ENDOMORPHISMS OF HYPERSURFACES
}

\author{
ILYA KARZHEMANOV \\ Abstract. For any prime $p \geq 5$, we show that generic hypersurface $X_{p} \subset \mathbb{P}^{p}$ \\ defined over $\mathbb{Q}$ admits a non-trivial rational dominant self-map of degree $>1$, \\ defined over $\overline{\mathbb{Q}}$. A simple arithmetic application of this fact is also given.
}

\section{INTRODUCTION}

1.1. Let $X$ be an algebraic variety (smooth, projective, over a field of characteristic 0). Then two groups of symmetries of $X$, namely $\operatorname{Aut}(X)$ (biregular automorphisms) and $\operatorname{Bir}(X)$ (birational ones), come for free. The question on whether these groups differ is classical and very important. For instance, the property $\operatorname{Aut}(X)=\operatorname{Bir}(X)$, signifying birational (super)rigidity of $X$, is an obstruction for $X$ to be rational (such $X$ had been studied in numerous papers including [16, 24], [9], 17] and [18]).

Typically one has $\operatorname{Aut}(X)=\{\mathrm{id}\}$ however. In this regard, it is natural to consider another (more general) class of symmetries of $X$, namely $\operatorname{End}(X)$, consisting of rational dominant endomorphisms of $X$. Then one may ask (what seems to be a folklore) whether $\operatorname{End}(X)=\operatorname{Bir}(X)$ ? (Note that the latter property is an obstruction for $X$ to be unirational.) This question gets immediate answer ('yes') when $X$ - with $\operatorname{Pic}(X)=\mathbb{Z}$ at least — is of general type (as the endomorphisms preserve the spaces $H^{0}\left(X, m K_{X}\right)$ for all $\left.m \in \mathbb{Z}\right)$. On the other hand, already in the Calabi-Yau case things are not that straightforward; although still one gets $\operatorname{End}(X)=\operatorname{Bir}(X)$ when $X$ is a general K3 surface for example (see [7).

Presently, we would like to treat rationally connected $X$, namely $X:=X_{N} \subset \mathbb{P}^{N}$ being a hypersurface of degree $N$ (see [24, [15, 6] for some other aspects of the geometry of these $X_{N}$ ). Recall that according to [10] every $X_{N}, N \geq 4$, is nonrational, having $\operatorname{Bir}\left(X_{N}\right)=\operatorname{Aut}\left(X_{N}\right)$.

Our main result is

MS 2010 classification: 14E05, 14D06, 14M20. 
Theorem 1.2. For any prime $p \geq 5$, (Zariski) general $\mathbb{Q}$-hypersurface $X_{p} \subset \mathbb{P}^{p}$ admits an endomorphism $f_{X_{p}}$ of degree $>1$, defined over $\overline{\mathbb{Q}}$. More precisely, $f_{X_{p}}$ has a field of definition - $\mathbf{k}$ below, - same for all $X_{p}$.

Thus the "non-regular" geometry of $X=X_{p}$ is pretty much fruitful (compare with results in [2, 4, 23], accounting for regular self-maps of $X$ ). At the same time, the proof of Theorem 1.2 is not effective, and it would be interesting to describe (a part of) $\operatorname{End}(X)$ explicitly. For example, what is the $\operatorname{End}(X)$-action on the universal Chow group of $X$ (cf. 25, 3])? It is also plausible to get rid of the degree/ground field/dimension assumption in the formulation of our result. Say, can one take any Fano manifold $X$ in place of $X_{p}$, or at least any (composite) integer $N$ instead of $p$ ?

1.3. In order to prove Theorem 1.2 we first relate $X_{p}$ to those hypersurfaces that have lots of endomorphisms. The latter are $X_{d} \subset \mathbb{P}^{N}$, defined over a given field, that happen to be unirational over this field, provided that $N \gg d$ is sufficiently large (see 2.1 below for the precise statements).

Next we employ the degree formula from [26] (cf. [22, 19], 14]). Recall that this formula relates the connective K-theory classes of two algebraic varieties $X$ and $Y$ admitting some rational map $f: X \rightarrow Y$. However, in order to get something fruitful in this way (e.g. to show that $f$ with $\operatorname{deg} f=0$ does not exist) one has to consider $X, Y$, etc. to be defined over an algebraically non-closed field $\mathbf{k}$, and (roughly speaking) to have no points over k. This is the reason for the degree (resp. dimension/ground field) restriction in Theorem 1.2 .

Remark 1.4. One of the basic obstructions for applying our method to arbitrary degree $d$ (Fano) hypersurfaces $X_{d} \subset \mathbb{P}^{N}$ is that those usually have points over the field of definition (cf. [5]). There may also be no such nice congruence relation as in Corollary 2.5 below. But still, once we find (sufficiently many?) endomorphisms of $X_{p} \subset \mathbb{P}^{p}$ which preserve some projective subspace $\Gamma \subset X_{p}$, one may hope (by projecting from $\Gamma$ ) to obtain non-trivial endomorphisms of $X_{d}$ for some (all?) $d \leq p-1$.

The principal part of our arguments relies on (a part of) the main result in 8 which asserts the existence of $X=X_{p}=Y$ as indicated above. It is then not hard to derive Theorem 1.2 for the given $X_{p}$ and the general case follows easily (see Section 3 for details). Again we indicate that the initial $X_{p}$ is very special. (It is 
defined over a field $\mathbf{k}$ of cohomological dimension $\leq 1$ and does not contain points over the extensions of $\mathbf{k}$ whose degrees are coprime with $p$.)

1.5. The next result was motivated by the paper [1]:

Corollary 1.6. Let $X_{p} \subset \mathbb{P}^{p}$ be as in Theorem 1.2. Then there exists a possibly larger number field $K \supseteq \mathbb{Q}$ such that Zariski closure of the $f_{X_{p}}$-orbit of the set $X_{p}(K)$ of $K$-rational points on $X_{p}$ has dimension $\geq 2$.

One may consider Corollary 1.6 as a generalization of [13, Theorem 1.4]. Yet, unfortunately, our conclusion is weaker and it would be interesting to establish potential density of the set $X_{p}(\mathbb{Q})$ in $X_{p}$ (e.g. by refining the arguments of Section 4 below).

Acknowledgments. I would like to thank F. Bogomolov and K. Zainoulline for their interest and fruitful conversations. The results of this paper have been discussed with K. Oguiso, S. Okawa, and B. Poonen during my visits to Osaka University and MIT in Spring 2015; I am grateful to these people for hospitality. Also comments by anonymous referee have helped to improve the exposition. The work was supported by World Premier International Research Initiative (WPI), MEXT, Japan, and Grant-in-Aid for Scientific Research (26887009) from Japan Mathematical Society (Kakenhi).

\section{Preliminaries}

2.1. Fix some integers $d$ and $r \geq 1$. Recall that every smooth hypersurface $X_{d} \subset \mathbb{P}^{N}$ of degree $d$ contains a projective subspace $\Lambda \simeq \mathbb{P}^{r}$ when $N \gg 1$ (see [12], [20]). More precisely, $X_{d}$ corresponds to (generic) point in the incidence subvariety

$$
Z:=\left\{\left(X_{d}, \Lambda\right) \mid \Lambda \subset X_{d}\right\} \subset \mathbb{P}^{\left({ }^{N+d}\right)} \times \operatorname{Gr}(r+1, N+1),
$$

with dominant projections $Z \longrightarrow \mathbb{P}$, Gr.

Further, if $\Lambda$ is given over an arbitrary field $\mathbf{k}_{0} \subset \mathbb{C}$ by equations $l_{N-r+1}=$ $\ldots=l_{N}=0$ for some linear forms $l_{i}$, then $X_{d}$ can be chosen to be defined over $\mathbf{k}:=\mathbf{k}_{0}(\sqrt{-1})$. Indeed, any $X_{d}$ passing through $\Lambda$ has the defining equation $\sum_{i=N-r+1}^{N} \phi_{i} l_{i}=0$, for some (varying) forms $\phi_{i}$ of degree $d-1$. Hence, since the set of k-points is obviously dense in $\mathbb{P}^{\left({ }^{N+d-1} d-1\right.}$ ) (w.r.t. the complex analytic 
topology), one can approximate $\phi_{i}$ by such $\mathbf{k}$-forms of degree $d-1$ that $X_{d}$ remains smooth.

Thus, since one can always find generic $\Lambda \in \operatorname{Gr}(r, N)$ to be defined over $\mathbf{k}$, the above discussion provides a smooth hypersurface $X_{d}$ and a projective subspace $\Lambda \subset X_{d}$, both over $\mathbf{k}$, for any given $d, r$ and sufficiently large $N=N(d, r)$. From [12, Corollary 3.7] we obtain

Theorem 2.2 (Harris, Mazur, Pandharipande). $X_{d}$ is k-unirational.

2.3. Let $X, Y$ be smooth projective geometrically irreducible $\mathbf{k}_{0}$-varieties of dimension $d=\operatorname{dim} X=\operatorname{dim} Y$. Assume that there is a rational $\mathbf{k}_{0}$-map $f: X \rightarrow Y$. The degree $\operatorname{deg} f$ equals 0 if $f$ is non-dominant; otherwise $\operatorname{deg} f:=\left[\mathbf{k}_{0}(X)\right.$ : $\left.f^{*} \mathbf{k}_{0}(Y)\right]$.

We recall the next result from [26]:

Theorem 2.4 (Zainoulline). $\chi\left(\mathcal{O}_{X}\right) \cdot \tau_{d-1} \equiv \operatorname{deg} f \cdot \chi\left(\mathcal{O}_{Y}\right) \cdot \tau_{d-1} \bmod n_{Y}$, where $\chi(\cdot)$ is the Euler characteristic,

$$
\tau_{d-1}:=\prod_{p \text { prime }} p^{\left[\frac{d-1}{p-1}\right]}
$$

is the $(d-1)$-st Todd number, and $n_{Y}$ is the g.c.d. of degrees of all closed points on $Y$.

An immediate consequence of Theorem 2.4 is

Corollary 2.5 (cf. [26, Example 6.4]). In the previous notation, if $n_{X}=p$, $X=Y=X_{p} \subset \mathbb{P}^{p}$ (i.e. $d=p-1$ and $N=p$ ) for some prime $p \geq 3$, and (more generally) $f$ is a k-map, then $\operatorname{deg} f>0$. (Such $X$ is called incompressible over k. 1)

Proof. Regard $X$ as a hypersurface over $\mathbf{k}$. Then, since $\left[\mathbf{k}: \mathbf{k}_{0}\right] \leq 2$, we get $n_{X}=p$ again. The claim now follows from Theorem 2.4 (with $\mathbf{k}_{0}$ replaced by $\mathbf{k}$ ) and the fact that $\chi\left(\mathcal{O}_{X}\right)=1=\left(\tau_{p-2}, p\right)$.

Existence of $X=X_{p}$ as in Corollary [2.5] is guaranteed by the following result (see [8, Theorem 8]):

1) Existence of similar hypersurfaces $X$ of general type, as suggested by [26, Example 6.4], is not clear in the current setting because the condition $n_{X}=p$ need not be satisfied (compare with Proposition 2.6 below). 
Proposition 2.6 (Colliot-Thélène). For every prime $p \geq 5$, there is a smooth (over $\overline{\mathbb{Q}})$ hypersurface $X \subset \mathbb{P}^{p}$, given (over $\mathbb{Q}$ ) by the equation

$$
x_{1}^{p}+l x_{2}^{p}+\ldots+l^{p-1} x_{p}^{p}-\alpha x_{0}^{p}=0
$$

for some integers $l, \alpha$, so that $n_{X}=p$.

Remark 2.7. Let $t$ be a transcendental parameter, $X_{d} \subset \mathbb{P}^{N}$ some $\mathbb{Q}(t)$ hypersurface, and $d=N=p$. One may assume (e.g. by identifying $X_{d}$ with an appropriate pencil of degree $d=p$ hypersurfaces) that this $X_{d}$ specializes via $t \mapsto 0$ to $X$ from Proposition 2.6. It follows then that generic such $X_{d}$ also satisfies $n_{X_{d}}=p$. (Here "generic hypersurface" means a point in a Zariski open subset of the $\mathbb{Q}(t)$-variety $\mathbb{P}^{\left({ }^{N+d}\right)}$ ) parameterizing all hypersurfaces of degree $d$ in $\mathbb{P}^{N}$ defined over the field $\mathbb{Q}(t)$.) Indeed, by definition of $n_{Y}$ (see Theorem 2.4) and specialization $t \mapsto 0$ one finds that $n_{X_{d}}$ divides $p=n_{X}$, and once $n_{X_{d}}=1$ we get (by the same reasoning) that $n_{X}=1$ as well, a contradiction.

\section{Proof of Theorem 1.2}

We keep on with notation of Section 2

3.1. Let $X$ be as in Proposition 2.6. Consider a cone $\widehat{X} \subset \mathbb{P}^{N}$ over $X$ of sufficiently large dimension and a family $\mathcal{X}$ of degree $p$ hypersurfaces $\left(\subset \mathbb{P}^{N}\right)$ over $\mathbb{Q}$ that smooths out the singularities of $\widehat{X}$. We may regard (the general fiber of) $\mathcal{X}$ as a smooth $\mathbf{k}_{0}$-hypersurface of degree $p$ in $\mathbb{P}^{N}$ for some purely transcendental field $\mathbf{k}_{0} \supset \mathbb{Q}$.

Next, using the (dominant) projections $Z \longrightarrow \mathbb{P}$, Gr one can see by the same argument as in 2.1 that the set of all k-points $\left(X_{p}, \Lambda\right) \in Z$ is dense on $Z$ in the complex analytic topology, and so $\mathcal{X}$ can be approximated by k-hypersurfaces $X_{p}$ (for $\mathcal{X}, X_{p}$ treated as points on $\mathbb{P}$, with $\mathbf{k} \subset \mathbb{C}$ ). The hypersurfaces $\mathcal{X}$ and $X_{p}$ can actually be put on an affine line $\mathbb{A}_{\mathbf{k}}^{1} \subset \mathbb{P}$ in such a way that the preimage $\widetilde{\mathbb{A}_{\mathbf{k}}^{1}} \subset Z$ of $\mathbb{A}_{\mathbf{k}}^{1}$ has all fibers $=$ some projective spaces and generic fiber isomorphic to $\Lambda$ (apply the reasoning from $2 \mathbf{2 . 1}$ to this family over $\mathbb{A}_{\mathbf{k}}^{1}$ considered as a degree $p$ hypersurface over $\left.\mathbf{k}_{0}(t)\right)$.

Lemma 3.2. In the previous setting, the hypersurface $\mathcal{X}$ contains a projective subspace $\subset \mathbb{P}^{N}$, isomorphic to $\Lambda$ and defined over $\mathbf{k}$. 
Proof. It suffices to show that there exists a k-point on $Z$ whose projection to $\mathbb{P}$ coincides with $\mathcal{X}$. Indeed, the preceding natural projection $\widetilde{\mathbb{A}_{\mathbf{k}}^{1}} \longrightarrow \mathbb{A}_{\mathbf{k}}^{1}$ is $\operatorname{Gal}(\overline{\mathbf{k}} /$ $\mathbf{k}$ )-equivariant, hence its fiber over $\mathcal{X}$ is a projective $\mathbf{k}$-space.

Without loss of generality we will assume that $\mathbf{k}_{0}=\mathbb{Q}(t)$ in what follows.

Lemma 3.3. There exists a $\mathbf{k}$-endomorphism $f: \mathcal{X} \rightarrow \mathcal{X}$ such that $\operatorname{deg} f>1$.

Proof. It follows from Lemma 3.2 and Theorem 2.2 (applied to $X_{d}:=\mathcal{X}$ ) that $\mathcal{X}$ is $\mathbf{k}$-unirational. This yields two rational dominant k-maps $\phi: \mathbb{P}^{N-1} \rightarrow \mathcal{X}$ and $\psi: \mathcal{X} \rightarrow \mathbb{P}^{N-1}$. We may assume one of $\phi, \psi$ to have degree $>1$. Then it remains to take $f:=\phi \circ \psi$.

3.4. Let $f$ be as in Lemma 3.3. Note that $f$ is induced by a rational self-map of $\mathbb{P}^{N} \supset \mathcal{X}, \widehat{X}$. Indeed, since $p \geq 5$ and thus $\operatorname{Pic}(\mathcal{X})=\mathbb{Z}$ (Lefschetz), the map $f$ is given by such a linear system on $\mathcal{X}$ that is obtained via restriction of a linear system from $\mathbb{P}^{N}$.

Put $f_{0}$ to be the specialization of $f$ at the fiber $\widehat{X}$ of the family $\mathcal{X}$ (recall that $\left.\mathbf{k}_{0}=\mathbb{Q}(t)\right)$. More precisely, if $\mathcal{L}_{t}$ is a (movable) linear system defining $f$, then its specialization $\mathcal{L}_{0}$ to $\widehat{X}$ may acquire some divisorial components in the base locus 2) Subtracting all these we arrive at a linear system which we set to define $f_{0}$.

Lemma 3.5. $f_{0}$ is not induced by a self-map of $\operatorname{Sing}(\widehat{X})$ (for an appropriate $f$ ). In other words, if $x_{0}=\ldots=x_{p}=0$ are the equations of the singular locus $\operatorname{Sing}(\widehat{X}) \subset \widehat{X}$, then $f_{0} \neq \mathrm{id}, 0$ on the subspace $\mathbb{P}^{p} \subset \mathbb{P}^{N}$ complementary to $\operatorname{Sing}(\widehat{X})$.

Proof. Let the notation be as in the proof of Lemma 3.3. One can choose a k-point $o \in \mathcal{X}$ such that $\psi($ resp. $\phi)$ is unramified at $o$ (resp. $\psi(o)$ ).

Take $f:=\phi \circ \sigma \circ \psi$ for some $\sigma \in \operatorname{PGL}(N, \mathbf{k})$ (to be specified further) in such a way that $f(o)=o$. Namely, since $\psi, \phi$ induce isomorphisms of the tangent spaces $T_{o}=T_{\phi(\psi(o))}, T_{\psi(o)}$, and (adjoint of) $\sigma$ can act transitively on the $N$-tuples of k-

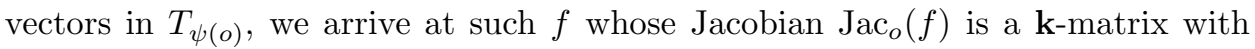
pairwise distinct eigen values and all defined over $\mathbb{Q}$. Then, considering $f_{0}$ as a rational self-map of $\mathbb{P}^{N}$ (cf. the discussion at the beginning of 3.4), we obtain that all non-zero eigen values of the matrix $\operatorname{Jac}_{o^{\prime}}\left(f_{0}\right)$ are pairwise distinct as well. Here $o^{\prime} \in \widehat{X}$ is the specialization of $o$

2) The latter stems from the fact that $f$ may not be defined (in codimension 2) on $\widehat{X}$.

3) Note that $o^{\prime}$ is not contained in the indeterminacy locus of $f_{0}$ for the indicated $o$ and $\sigma$. Thus $f_{0}$ is defined at $o^{\prime}$ and satisfies $f_{0}\left(o^{\prime}\right)=o^{\prime}$. 
Now suppose that $f_{0}$ is induced by some rational endomorphism of $\operatorname{Sing}(\widehat{X})$. Again we regard $f_{0}$ as a self-map of $\mathbb{P}^{N}$. Then $f_{0}$ is regular at some point $o^{\prime} \in \mathbb{P}^{N}$, $f_{0}\left(o^{\prime}\right)=o^{\prime}$ and the non-zero eigen values of $\operatorname{Jac}_{o^{\prime}}\left(f_{0}\right)$ are all different. On the other hand, $f_{0}=$ id on the subspace $\mathbb{P}^{p} \subset \mathbb{P}^{N}$ complementary to $\operatorname{Sing}(\widehat{X})$, a contradiction.

Same argument shows that $f_{0}$ has non-trivial components on $\mathbb{P}^{p}$, i. e. $f_{0} \neq 0$ there, which concludes the proof.

Note that generic subspace $\mathbb{P}^{p} \subset \mathbb{P}^{N}$ cuts out a subvariety on $\widehat{X}$ isomorphic $X$ (cf. the beginning of (3.1). Identify $X$ with such a section $\mathbb{P}^{p} \cap \widehat{X}$ so that the restriction $\left.f_{0}\right|_{X}$ is a well-defined rational map. Let $f_{X}: X \rightarrow X$ be the composition of $\left.f_{0}\right|_{X}$ and the linear projection $\widehat{X} \rightarrow X$ from $\operatorname{Sing}(\widehat{X})$.

Lemma 3.6. $f_{X} \neq$ id and is dominant.

Proof. Indeed, $f_{0}$ induces a non-identical map on $X$ by Lemma 3.5, and it remains to apply Corollary 2.5 to the $\mathbb{Q}(\sqrt{-1})$-map $f_{X}$.

Remark 3.7. For the last part of Lemma 3.6, observe that the fact $\operatorname{deg} f_{X} \neq 0$ is not immediate from the proof of Lemma 3.5, and hence one needs an additional argument (results of the second half of Section 2 for instance) in order to proceed.

Theorem 1.2 (for the given $X$ ) now follows from

Proposition 3.8. $\operatorname{deg} f_{X}>1$.

Proof. Regard $\mathcal{X}$ as a (flat) family of hypersurfaces $\mathcal{X}_{t} \subset \mathbb{P}^{N} \times t \subset \mathbb{P}^{N} \times \mathbb{P}^{1}$ (with $\mathcal{X}_{0}:=\widehat{X}$ ). Let $\pi: \mathcal{X} \longrightarrow \mathbb{P}^{1}$ be the natural projection (so that $\pi^{-1}(t)=\mathcal{X}_{t}$ ). Let also $\mathcal{L}_{t}$ be as in the second paragraph of $\mathbf{3 . 4}$

Lemma 3.9. The linear system $\mathcal{L}_{0}$ is non-trivial on $\widehat{X}$ (unless $\operatorname{deg} f_{X}>1$ ).

Proof. Suppose the contrary (i. e. $\mathcal{L}_{0}=\{0\}$ ). Then the $\pi$-map $f: \mathcal{X} \rightarrow \mathcal{X}$ has indeterminacies along $\widehat{X}$. Resolve these by some $\pi$-blow-up $\sigma: \mathcal{Y} \longrightarrow \mathcal{X}$. Let $\mathcal{Y} \stackrel{a}{\longrightarrow} \mathcal{Z} \stackrel{b}{\longrightarrow} \mathcal{X}$ be the Stein factorization of the resolved $f$. Here $a, b$ are some $\pi$ morphisms, with $a$ inducing a birational isomorphism between $\mathcal{Y}$ and $\mathcal{Z}$. Moreover, since $f$ is not defined on $\widehat{X}$, the proper transform $\sigma_{*}^{-1} \widehat{X}$ of $\widehat{X}$ on $\mathcal{Y}$ belongs to the exceptional locus of $a$. Composing further with $b$ yields a rational self-map $\mu: \widehat{X} \rightarrow \widehat{X}$ of degree 0 .

Notice that $\mu$ does not coincide with the projection $\widehat{X} \rightarrow X$ from $\operatorname{Sing}(\widehat{X})$ because $\operatorname{deg} b>1$ (cf. Lemma 3.3) and $\mathcal{X}_{0}=\widehat{X}$ is a non-multiple fiber of $\pi$ (so that 
$\widehat{X}$ is not a branching divisor of $b$ ). Thus one may assume $X=\mathbb{P}^{p} \cap \widehat{X}$ intersects generic fiber of $\mu$ at $>1$ points. Composing $\left.\mu\right|_{X}$ with $\widehat{X} \rightarrow X$ either gives a selfmap $X \rightarrow X$ of degree $>1$ (which we take for $f_{X}$ ), or that $X$ is not incompressible, in contradiction with Corollary 2.5,

Let $\mathcal{Y}, \sigma, a, \ldots$ be as above. It follows from Lemma 3.9 that $\widehat{X}=\mathcal{X}_{0}$ and the scheme $\mathcal{Z}_{0}:=b^{-1}(0)$ are birationally isomorphic via $a \circ \sigma^{-1}$ (recall that $b$ is finite). In particular, $\mathcal{Z}_{0}$ is not a ramification divisor of $b$, which implies that $\left.\operatorname{deg} b\right|_{\mathcal{Z}_{t}}=$ $\left.\operatorname{deg} b\right|_{\mathcal{Z}_{0}}$ for all $t \in \mathbb{C}$ close to 0 .

Now, since $\left.\operatorname{deg} b\right|_{\mathcal{Z}_{t}}>1$ by construction, we deduce that $\operatorname{deg} f_{0}=\left.\operatorname{deg} b\right|_{\mathcal{Z}_{0}}>1$ as well. The latter also gives $\operatorname{deg} f_{X}>1$. Indeed, otherwise restricting $\mathcal{L}_{0}$ to generic $X=\mathbb{P}^{p} \cap \widehat{X}$, we get $f_{X} \in \operatorname{Aut}(X)$ according to Lemma 3.6 and [10]. But then, since $f_{X}$ is composed of $\left.f_{0}\right|_{X}$ and projection $\widehat{X} \rightarrow X$ to the base of the cone, we obtain that $f_{0}$ must be induced by some projective transformation of $\mathbb{P}^{N} \supset \widehat{X}$. The latter obviously contradicts $\operatorname{deg} f_{0}>1$ and the proof of Proposition 3.8 is finished.

To complete the proof of Theorem 1.2 we simply apply Remark 2.7 and the fact that all the preceding arguments go verbatim with $\mathbb{Q}$ replaced by $\mathbb{Q}(t)$. It remains to set $t:=t_{0} \in \mathbb{Q}$ - a given general parameter value - to obtain hypersurfaces as in the statement of Theorem 1.2. Furthermore, in the generic setting one can replace the argument with $f_{X} \in \operatorname{Aut}(X)$ at the end of the proof of Proposition 3.8 by that with $f_{X}=$ id (cf. [21, Theorem 5]), thus getting a contradiction with Lemma 3.6 4)

\section{Proof of Corollary 1.6}

4.1. We proceed with applying the constructions of Section 3 to study the arithmetics of hypersurfaces $X_{p} \subset \mathbb{P}^{p}$ (the notation is as earlier) 5)

Let again $X$ be as in Proposition 2.6

Lemma 4.2. $f_{X}$ is non-periodic.

\footnotetext{
4) This argument may be considered as another way to prove Theorem 1.2

5) Note that one can not specialize the (abundance of) k-points on $\mathcal{X}$ to "many" $\mathbb{Q}$-points on the cone $\widehat{X}$ (as we did with (some of) endomorphisms) because $n_{X}=p$ and so all the points on $\widehat{X}$ we obtain this way are concentrated on $\operatorname{Sing}(\widehat{X})$.
} 
Proof. Indeed, otherwise we have $f_{X}^{k}=$ id for some $k$, so that both $f_{X}, f_{X}^{k-1}$ are invertible. But this contradicts Proposition 3.8 .

Fix $f_{X}$ as in Lemma 4.2. Then after possibly replacing $f_{X}$ by $f_{X}^{k}, k \gg 1$, we obtain a point $o \in X(K)$ such that $f_{X}(o)=o$ and $f_{X}$ is defined at $o$ (see [1]). We also have $\operatorname{det} \operatorname{Jac}_{o}\left(f_{X}\right) \neq 0$ because $f_{X}$ is dominant.

Lemma 4.3. There exists a $\mathbf{k}$-cube $\square_{o} \subset X$ (i.e. $\square_{o}$ is given by linear inequalities with coefficients in $\mathbf{k}$ ) centered at $o$ and invariant under $f_{X}$.

Proof. Let $\square \subset X$ be some cube containing $o$ and defined over $\mathbb{Q}$. Then the set $\bigcup_{k>0} X \backslash f_{X}^{k}(\square)$ is not everywhere dense in $X$. Indeed, otherwise there would exist a subsequence $f_{X}^{k_{i}}(\square) \rightarrow o$ for $i \rightarrow \infty$, which implies that $o \in X(\mathbb{Q})$ and contradicts $n_{X}=p$. It remains to take $f_{X}$-invariant $\square_{o} \subseteq \bigcap_{k>0} f_{X}^{k}(\square)$.

It follows from Lemma 4.3 (and the implicit function theorem) that the eigen values $\lambda_{i}$ of the matrix $\operatorname{Jac}_{o}\left(f_{X}\right)$ are algebraic numbers (from $K$ ), all having norms $\left|\lambda_{i}\right|=1,1 \leq i \leq p-1$. Furthermore, making if necessary a coordinate change on $\mathbb{P}^{p} \supset X$ of the form $x_{j} \mapsto \alpha_{j} x_{j}, 0 \leq j \leq p$ (i.e. rescaling the metric on $X$ ), for some $\alpha_{j} \in \mathbb{Q}^{*}$, we may assume $\lambda_{i}$ to be algebraic integers (from $O_{K}$ ).

Lemma 4.4. The matrix $\operatorname{Jac}_{o}\left(f_{X}\right)$ is semi-simple. Moreover, there is $j<p-2$ such that $\lambda_{1}=\ldots=\lambda_{j}= \pm 1$, while $\lambda_{j+1}, \ldots, \lambda_{p-1}$ are multiplicatively independent.

Proof. The first claim follows from the fact that $f_{X}$ linearizes on $\square_{o}$. Now, if $\lambda_{i}= \pm 1$ (or equivalently $\lambda_{i} \in \mathbb{R}$ ) for all $i$, then $f_{X}^{2}=\mathrm{id}$, a contradiction.

Further, Dirichlet's unit theorem yields $\lambda_{j+1} \ldots \lambda_{p-1}=\left|\lambda_{j+1} \ldots \lambda_{p-1}\right|=1, \lambda_{j}=$ $\lambda_{k}^{-1}$ as the only relations between $\lambda_{j+1}, \ldots, \lambda_{p-1} \in O_{K}^{*}$. In particular, since $j<$ $p-1, \lambda_{j+1}, \ldots, \lambda_{p-1}$ generate a free subgroup $\subseteq \mathbb{Z}^{p-j-1} \subset O_{K}^{*}$.

Suppose that $j=p-2$. Then $\lambda_{j+1}, \ldots, \lambda_{p-1}$ are all equal to $\lambda_{j+1}^{ \pm 1}$ say. On the other hand, characteristic polynomial of $\operatorname{Jac}_{o}\left(f_{X}\right)$ is defined over $\mathbf{k}$ (by construction of $\square_{o}$ in Lemma 4.3), and hence the minimal polynomial of $\lambda_{j+1}$ divides it. All together, this implies that $\lambda_{i}^{2}= \pm 1$ for all $i \geq j+1$, a contradiction.

Thus we get $j<p-2$ and the claim follows.

The arguments in [1, Section 2] and Lemma 4.4 imply that Zariski closure of the $f_{X}$-orbit of the locus $X(K)$ has dimension $\geq p-j-1>1$. 
Finally, to complete the proof of Corollary 1.6 one replaces $\mathbb{Q}$ by $\mathbb{Q}(t)$, as at the end of Section 3, and repeats the previous arguments.

\section{REFERENCES}

[1] E. Amerik, F. Bogomolov and M. Rovinsky, Remarks on endomorphisms and rational points, Compos. Math. 147 (2011), 1819 - 1842.

[2] E. Amerik, M. Rovinsky and A. Van de Ven, A boundedness theorem for morphisms between threefolds, Ann. Inst. Fourier (Grenoble) 49 (1999), 405 - 415.

[3] A. Auel, J.-L. Colliot-Thélène and R. Parimala, Universal unramified cohomology of cubic fourfolds containing a plane, Preprint arXiv:1310.6705

[4] A. Beauville, Endomorphisms of hypersurfaces and other manifolds, Internat. Math. Res. Notices 1 (2001), $53-58$.

[5] B. J. Birch, Forms in many variables, Proc. Roy. Soc. Ser. A 265 (1961/1962), 245 - 263.

[6] I. Cheltsov and I. Karzhemanov, Halphen pencils on quartic threefolds, Adv. Math. 223 (2010), $594-618$.

[7] X. Chen, Self rational maps of K3 surfaces, Preprint arXiv:1008.1619, 2010.

[8] J.-L. Colliot-Thélène, Fields of cohomological dimension 1 versus $C_{1}$-fields, in Algebra and number theory, Hindustan Book Agency, Delhi, 2005, 1 - 6.

[9] A. Corti, Singularities of linear systems and 3-fold birational geometry, in Explicit birational geometry of 3-folds, London Math. Soc. Lecture Note Ser., 281, Cambridge Univ. Press, Cambridge, 2000, 259 - 312.

[10] T. de Fernex, Birationally rigid hypersurfaces, Invent. Math. 192 (2013), 533 - 566.

[11] N. Fakhruddin, Questions on self maps of algebraic varieties, J. Ramanujan Math. Soc. 18 (2003), $109-122$.

[12] J. Harris, B. Mazur and R. Pandharipande, Hypersurfaces of low degree, Duke Math. J. 95 (1998), $125-160$.

[13] J. Harris and Y. Tschinkel, Rational points on quartics, Duke Math. J. 104 (2000), 477 500.

[14] O. Haution, Degree formula for the Euler characteristic, Proc. Amer. Math. Soc. 141 (2013), 1863 - 1869.

[15] V. A. Iskovskikh, Uspekhi Mat. Nauk 56 (2001), 3 - 86; translation in Russian Math. Surveys 56 (2001), $207-291$.

[16] V. A. Iskovskih and Yu. I. Manin, Three-dimensional quartics and counterexamples to the Lüroth problem, Mat. Sb. (N.S.) 86 (1971), $140-166$.

[17] V. A. Iskovskikh and A. V. Pukhlikov, Birational automorphisms of multidimensional algebraic manifolds, J. Math. Sci. 82 (1996), 3528 - 3613. 
[18] J. Kollár, Tr. Mat. Inst. Steklova, Mnogomernaya Algebraicheskaya Geometriya, 264 (2009), 103 - 108; translation in Proc. Steklov Inst. Math. 264 (2009), 96 - 101.

[19] J. Kollár, Esnault-Levine-Wittenberg indices, Preprint arXiv:1312.3923

[20] J. Kollár et al., Flips and abundance for algebraic threefolds, vol. 211, Astértisque, 1992.

[21] H. Matsumura and P. Monsky, On the automorphisms of hypersurfaces, J. Math. Kyoto Univ. 3 (1963/1964), 347 - 361.

[22] A. Merkurjev, Algebraic oriented cohomology theories, in Algebraic number theory and algebraic geometry, Contemp. Math., 300, Amer. Math. Soc., Providence, RI, 2002, 171 - 193.

[23] K. H. Paranjape and V. Srinivas, Self-maps of homogeneous spaces, Invent. Math. 98 (1989), $425-444$.

[24] A. V. Pukhlikov, Birational automorphisms of Fano hypersurfaces, Invent. Math. 134 (1998), $401-426$.

[25] C. Voisin, Unirational threefolds with no universal codimension 2 cycle, Invent. Math. 201 (2015), $207-237$.

[26] K. Zainoulline, Degree formula for connective $K$-theory, Invent. Math. 179 (2010), 507 - 522.

Kavli IPMU (WPI), The University of Tokyo, 5-1-5 Kashiwanoha, KASHIWA, 277 - 8583, JAPAN

Moscow Institute of Physics and Technology, 9 Institutskiy Per., Dolgoprudny, Moscow Region, 141701, Russia

E-mail address: ILYA.KARZHEMANOV@IPMU.JP 